\title{
Free Space Optics Vs Radio Frequency Wireless Communication
}

\author{
Rayan A. Alsemmeari and Sheikh Tahir Bakhsh \\ Faculty of Computing and Information Technology, King Abdulaziz University, Saudi Arabia \\ E-mail: \{ralsemmeari, stbakhsh\}@kau.edu.sa \\ Hani Alsemmeari \\ Institute of Public Administration Information and Technology department \\ E-mail: alsemmeariH@ipa.edu.sa
}

\begin{abstract}
This paper presents the free space optics (FSO) and radio frequency (RF) wireless communication. The paper explains the feature of FSO and compares it with the already deployed technology of RF communication in terms of data rate, efficiency, capacity and limitations. The data security is also discussed in the paper for identification of the system to be able to use in normal circumstances. These systems are also discussed in a way that they could efficiently combine to form the single systemwith greater throughput and higher reliability.
\end{abstract}

Index Terms-Free Space Optics, Radio frequency, Wireless communication, Data security.

\section{INTRODUCTION}

For wireless communication RF and FSO is both used in the practical field. Free space optics is not the new technology but has its deep root in the past to be used in wars. Each technology is used according to it ability and requirement in the particular sector. It is cheap technology in terms of its installation and could be installed in the areas very easily where no large setup for data communication could be installed. RF is limited to the bandwidth constraint but for the lower data rate RF is suitable for the long distance communication [1]. In free space optics the data rate changes and could be used where the line of sight is not a problem for the communication links. Secondly data security and performance are also the parameter on which systems are measured [2]. FSO provides better security condition than the RF links and is discussed further in the report. Free space optics is used in combination with fiber optic technology to maintain the data rate of system. Radio frequency communication is also used with fiber optic technology to enhance the data rate.

Free space optics technology is based on the line of sight optical beam for data communication [3, 4]. It is the actually the combination of both wireless technology and optical technology. The core of technology is invisible beam of light that provides optical bandwidth for transmission that includes the data of voice, information and video transmission. Optical communication is very old technology used in the past for signal communication but on very low data rates. Laser technology enhanced the use of free space optics and is now highly dependent on the laser technology. FSO in orig inal form was developed by the NASA and used for the military purposes in different era as fast communication link. The technology has many commonalities with the fiber optics technology but behaves differently in the field due to the method of transmission for both the technologies [5, 6].

$\mathrm{RF}$ technology is very old technology for communication. It is the wireless technology for data communication. It is considered to be in use for more than 100 years. In 1901 Marconi achieved his first successful data transmission using the RF signal from one remote station to other. Originally the band of $\mathrm{RF}$ is based on the very low frequency of kilo Hertz to $1 \mathrm{GHz}$. It can be further extended to different frequency range for microwave communication. At the initial phase radio frequency communication was just limited to the lower frequency band for the data transmission and was used for the purpose of radio and some military applications [7]. But with the passage of time the frequency range extended and increased into hundreds of Giga hertz due to the require ment of higher data rate and noise immun ity in the field. Different coding techniques also involved in order to make data secure and for efficient transmission. Frequency was also increased in order to compact the devices in terms of antenna length as length of antenna is dependent on the frequency rate $[8,9]$. Different RF link networks are used in the field and listed in table las below:

Table 1. Radio Frequency Technologies

\begin{tabular}{|c|c|c|c|c|}
\hline Networks & Technology & Connectivity & Data rate & Range \\
\hline WAN & $\begin{array}{c}\text { GSM } \\
\text { GPRS }\end{array}$ & $\begin{array}{c}\text { Mobile to } \\
\text { mobile }\end{array}$ & $\begin{array}{c}10 \mathrm{k}- \\
2.4 \mathrm{Mb} / \mathrm{s}\end{array}$ & Global \\
\hline MAN & $\begin{array}{c}802.16 \\
802.16 \mathrm{a} \\
802.16 \mathrm{e}\end{array}$ & $\begin{array}{c}\text { PC-HSWL } \\
\text { internet }\end{array}$ & $268 \mathrm{Mb} / \mathrm{s}$ & $50 \mathrm{~km}$ \\
\hline LAN & $\begin{array}{c}802.1 \mathrm{~b} \\
802.1 \mathrm{a} \\
802.1 \mathrm{~g}\end{array}$ & PC-PC & $\begin{array}{c}11- \\
54 \mathrm{Mb} / \mathrm{s}\end{array}$ & $100 \mathrm{~m}$ \\
\hline PAN & $\begin{array}{c}\text { Bluetooth } \\
\text { UWB }\end{array}$ & $\begin{array}{c}\text { Device to } \\
\text { system }\end{array}$ & $1-2 \mathrm{Mb} / \mathrm{s}$ & $10 \mathrm{~m}$ \\
\hline
\end{tabular}


The rest of the paper is organized as follows. Section II discusses the overview of FSO and RF system. The performance comparis on of FSO and RF is discussed in Section III. The performance of Hybrid system and its impact is explained in Section IV. Finally, the paper conclusions are drawn in Section V.

\section{OVERVIEW OF FSO AND RF SYSTEM}

The FSO system is based on the wireless link between two wireless units called optical transmitter and receiver for one way communication and optical transceivers for both side communication mostly used in the field. The transmitter is made up of an optical source which transmits light and a lens that directs this light signal into the atmosphere. The receiver is also connected with lens to sense incoming data and routed using fiber optic cable [10]. The data pulses are transmitted from trans mitter in the form of beams with conical shape. The transmitter laser side should be in line of sight with the receiver. ALL the link heads should be in line of sight for the successful transmission. Fig. 1 shows that the transmitter is in line of sight with the receiver and it is actually the first requirement of successful data transmission in free space optical communication. Although if the line of sight is disturbed to the certain extent than signal data could still be sent over the link but for higher data rate achievement and low error rate line of sight is critical requirement.

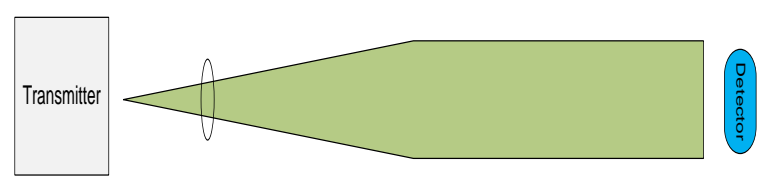

Fig. 1. Transmitter receiver alignment

As shown in Fig. 2 that different transmitters and receivers are attached in the network all transmitter and receiver are in line of sight and after receiving the data from one receiver it could be transmitted to the local network either consisting of fiber optic or some other networking media signals are passed on from one local network to other using free space optical equipment. Below mentioned block are showing how data is passed from one integral part of the system to another and all these major subsystems are combined in one unit to make system working. First the data is modulated on the high frequency signal with certain modulation scheme after that modulated data is passed on to the driver section which is linked with the laser part producing laser light according to the data provided after that signal is transmitted in the form of laser from transmitting optic. On the receiving side signal is gathered and same process occurs in reverse to take data out from the modulated signal. Other subsystems are installed in the unit to control different parameters of such system as direction etc.

RF system is wireless system based on the transmitting and receiving devices. Initially system was based on radio transmitters consisting of huge equipment installed in the building and receivers were radios carried by the common persons. The transmitter could be split into two devices as controller and Base station; they could be either combined or separated [11]. In case if base station is separate it acts as the link between the controller and remote receiver. Due to the advent of mobile communication the system is enhanced into the form that mobile receiver is turned into transceiver and can also connect the base station using the RF link.

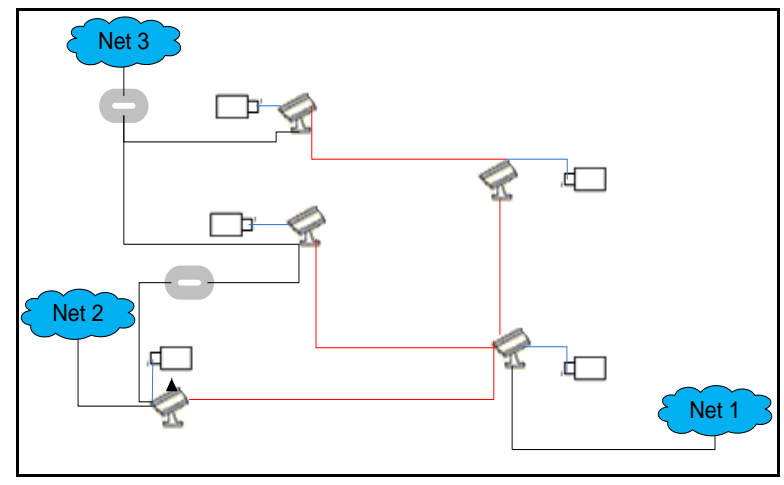

Fig.2. Atmospheric Laser communication

\section{PERFORMANCE COMPARISION OF FSO AND RF}

The following section provides detail analysis between FSO and RF.

\section{A. FSO Performance}

To observe the performance of free space optical system it is to observe lot of parameters of practical field. These parameters are divided in to two major sections one is the external parameter and the second one is internal parameters of the system. Internal parameters are completely related with the internal system specifications which includes the operating frequency of the system, power used by the system, divergence and angle of transmission. Similarly on the receiver side the capability of lens, its viewing capability and bit error rate [12]. Other parameters are external and not related to the system. These parameters include the environmental parameters and include following parameters:

$>$ Weather condition

$>$ Alignment

$>$ Atmospheric attenuation

$>$ Scintillation

$>$ Window attenuation

Performance of free space optical system is dependent on the weather conditions if the weather conditions are not clear and effect visibility in the open environment than the performance of system would be inversely affected. Typical free space optical system can operate 23 times greater than the naked eye capability. FSO systems transmit directional narrow beams of light and for receiving these beams of lights and giving good 
performance receiver must be exactly in alignment with the transmitter [13]. The starting beam diameter of typical FSO system starts with $5-8 \mathrm{~cm}$ and after passing through the field it goes up to $1-5 \mathrm{~m}$ over the range of $1 \mathrm{~km}$ as shown in Fig. 3.

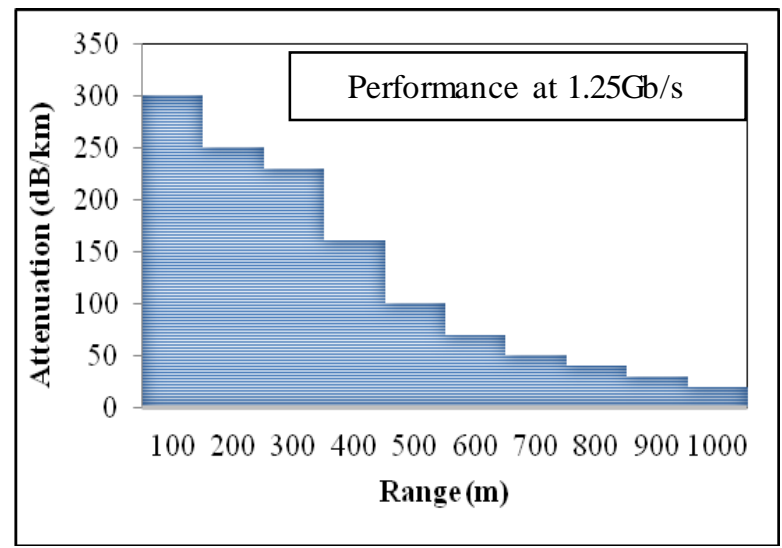

Fig.3. Attenuation Vs Range

It is not thinkable that constant buildings are in continuous motion due to certain factors and can affect the align ment and resultantly the performance of system. These conditions are thermal expansion and vibration. If the data rate is decreased the range of transmission could be increased at the particular bit error rate. Above graph show that if data rate is decreased from $1250-100 \mathrm{Mb} / \mathrm{s}$ the distance could be increased about 30 meters with attenuation of $200 \mathrm{~dB} / \mathrm{km}$. During the day time the intensity of light continuously changes and change in the light intensity also affect the performance of free FSO system in a way that thermal refraction of air changes whole this time that affects receiver sensitivity. But at the range of less than a $\mathrm{km}$ these scintillation affects are less harmful to system performance. The visibility condition of FSO system according to weather condition and distance are shown in Fig. 4.

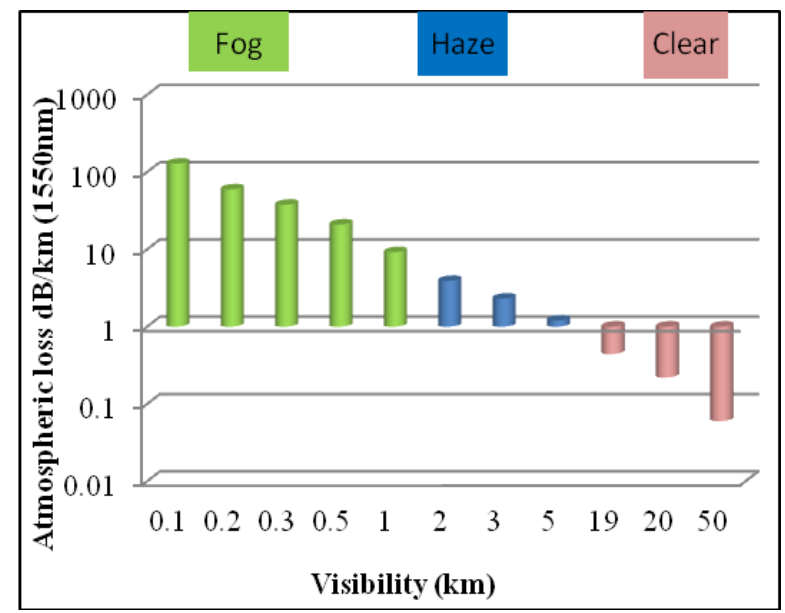

Fig.4. Atmospheric loss Vs visibility

\section{B. RF Performance}

There are many dynamic aspects of the radio frequency system performance. Performance of RF varies from system to system. There are many system used in the field like GSM, Bluetooth, GPRS etc. Their performance is dependent on the weather conditions as well as on the distance and also on the number of users using the system. The example of one of the system is broadband access. In this system the channel bandwidth is shared between the numbers of users. They share the capacity of channel and if the number of users increased the data rate goes low and when the number of users decrease in any point of time the whole bandwidth is shared between the users using the system. Signal strength of radio frequency communication system is actual strength of the system as radio waves travels long way for communication.

Other performance factors include availability of exact sites according to cell configuration. It is great possibility that if certain site required for base station might not be available for the installation and the performance of system is dependent on that location. So performance of system is compromised due to the location. Performance of RF system is also affected by the interference of electromagnetic signals and different noises. This is not the case in free space optic communication where everything is involved in the line of sight [13]. Still RF technology is considered to be one of the best technologies for communication due to long range communication and strong network on the earth the data could be sent from one place to another within seconds. The technology has strong footing and deep roots in our society in terms of performance and availability.

\section{FSO Throughput}

Free space optics technology is becoming the way to access broadband. Market is actually searching for the technology that could give them maximu m bandwidth so the data could be transmitted on the higher rates. Free space optics is very similar to the fiber optics in terms of data rate [14]. As the technology works on the same principle of transmitter and receiver with only difference of path where data is traveled from one point to the other saving not only the cost of fiber optic but also capable to achieve maximum efficiency. Throughput of any free space optics systems is heavily dependent on the weather conditions. If weather conditions are clear than with proper installation of equipment maximum throughput could be achieved. By using low cost led the data rate of 100 mega bit per second could be achieved easily. Some companies (Siemens) have claimed that they have achieved data rate of over $500 \mathrm{Mbit} / \mathrm{s}$. But many experiments have been conducted with $100 \mathrm{~GB} / \mathrm{s}$ fiber backhaul in the short range communication and they achieved data rate of 100 Giga bits per channel. In several practical application this system is being used and system exchanges the data rate of $10 \mathrm{~GB} / \mathrm{s}$. one of the application using this much data rate is high speed trains but the distance is only of few kilometers. Different products available in the market for short range communications between the buildings are operating at the data rate of $10 \mathrm{Mb} / \mathrm{s}$ to $2.5 \mathrm{~GB} / \mathrm{s}$ up to $4 \mathrm{~km}$ of the distance. 


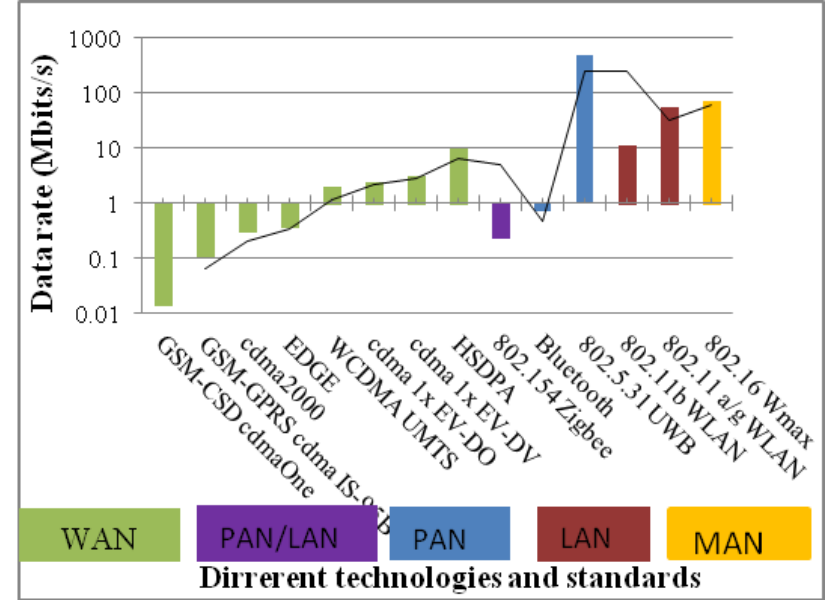

Fig.5. Technologies Vs data rates

\section{RF Throughput}

Radio communication is the key technology of all time and is now growing in every field of life either it is broadcasting or mobile communication etc. it could be split into different categories depending upon the use and data rate [15]. Also the receiving equipment is dependent on the data rate that it could handle. Lower data rates ensure better sensitivity towards the receiver side and so signal could be sensed in the long range. RF system data rate is dependent on the bandwidth given to the system and link distance. To increase the data rate the band width should be increased and the distance should be less. The range of RF frequency starts from $3 \mathrm{kHz}$ to $300 \mathrm{GHz}$. Throughput of RF versus FSO is defined in the Fig. 6 and clearly shows that within the 300 meter range the data rate of FSO is more than RF link.

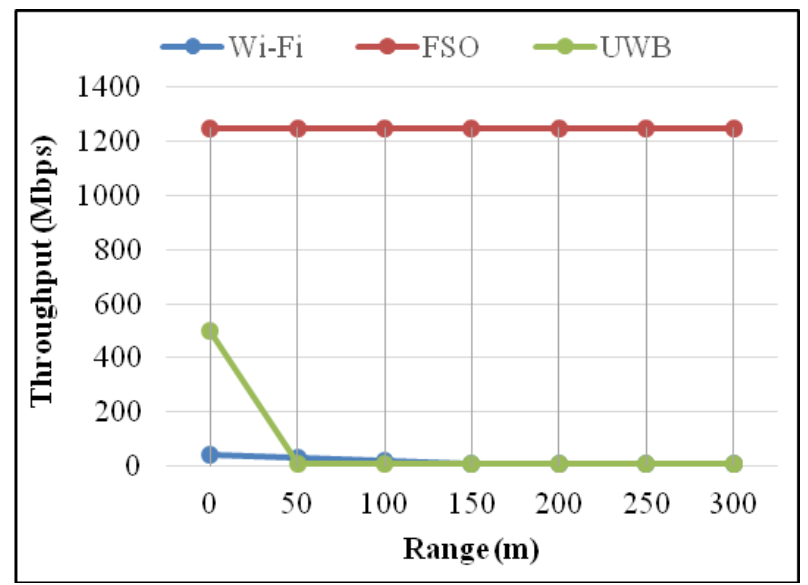

Fig.6. Through Vs range

\section{E. FSO Limitations}

FSO line of sight is great limitation for data transmission. So before installation of the equipment site survey is done according to the particular requirement for line of sight. Weather conditions produce strong limitations on the performance of free space optics. In case of heavy wind and fog in the environ ment affects the visibility and could decrease it tremendously low [16].
The visibility is important for lenses installed on the receiver side. If the visibility would get low the error rate on the receiving side would increase and the performance of system would go quite low. Another important thing is the sway of buildings that could cause the disturbance in the line of sight, and if the transmitter and receiver does not have the capability of adjusting the angle than the affect would be negligible.

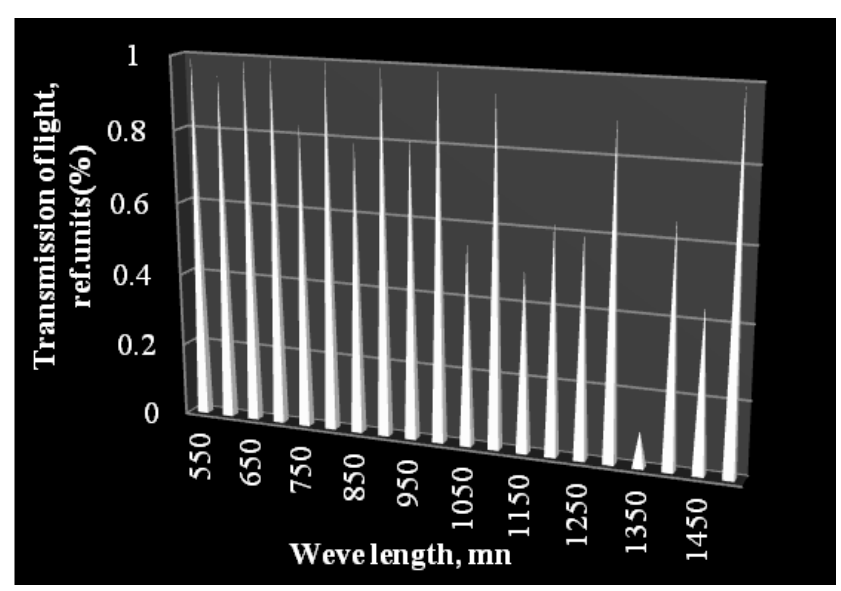

Fig.7. Attenuation of Laser Vs wavelength

Another limitation in the free space optics system is distance. As the distance between transmitter and receiver increases over the certain threshold the data rate decreases or even the chance of no reception. So this is the major bottleneck in the current systems.

\section{F. RF Limitation}

$\mathrm{RF}$ communication is the most famous wireless communication method used all over the world and currently preferred over the free space optical communication. Both systems have their own limitations. If we take optical communication its bandwidth is unregulated whereas RF system requires licensing in most of bands for its operation. Equipment of free space optics is cheaper than the RF commun ication system. RF system requires huge space to install the equipment. In terms of distance and data rate RF equipment requires more power and space $[17,18]$. Weather condition effects signals of both RF and free space optical systems.

Choosing the absolute weather conditions and applying the conditions of all parameter working correctly the theoretical maximum limit of free space optics could not be achieved. Maximum of $1 \mathrm{Gbit} / \mathrm{s}$ data rate at $500 \mathrm{~m}$ is assumed to be achievable under the absolute weather circumstance with $200 \mathrm{db} / \mathrm{km}$. in certain situation where huge amount of data is required by the system RF system cannot provide resource with such limited licensed spectrum range. So it is only the free space optical technology that can provide virtually unlimited data and secure data link. RF links require no line of sight for the communication the waves can travel through complex paths and with the range of base station the user can send and receive the data easily although the strength of signal is reduced due to the high building and dense environment but still applicable without line of sight. 
With respect to RF link they are low power and can give high data rate without interference with the RF links.

\section{G. Frequency Band}

In the early 1980 the only operation frequency band for the RF signaling and data networking was $450 \mathrm{MHz}$. the band width was quite lower and support only few kilo bits of data. This band of frequency was considered to be narrow band with limited data rate. After that spread spectrum came with enhancement in the band of frequency form $902 \mathrm{MHz}$ to $5.85 \mathrm{GHz}$. This band of frequency allowed the user to get efficient data transmission and reduced interference with other frequency bands [13]. User could achieve the data rate of almost $54 \mathrm{Mb} / \mathrm{s}$ within the range of almost 300 meters with almost 0.1 watt of power. In optical communication very wide band of frequency is available for the communication and data rate could be achieved up to the $\mathrm{Gb}$ it/s. The spectrum for FSO starts near the visible light and it operates in the range of $550 \mathrm{~nm}$ to $1500 \mathrm{~nm}$ wavelength. The frequency for this range goes in THz.

Wavelength of signal is dependent on the frequency of the signal. If frequency is less wave length will be higher. And waves with higher wavelength travel more distance than waves with smaller wave length. Both RF and FSO are wireless communication methods having their own advantage and disadvantages in certain circu mstances. RF is the best method of communication at lower data rates because its receivers are more sensitive to the radio wave that have scattered and diffracted different hurdles in the pathway. Channels are independent of being blocked by the obstacles in the field. At higher data rates frequency should be greater and for the higher frequency the line of sight becomes critical requirement for error free communication. So at the higher data rate free space optics becomes the promising technology for the communication and even terabits of data rate has been achieved in the lab experiments. As shown in table 2 the features that FSO is quite better than the RF wireless communication in almost every aspect whether its data rate power loss and security. Environmental conditions do affect the system but other features overcome this issue in terms of its efficiency.

\section{Table 2. RF Vs FSO}

\begin{tabular}{|c|c|c|}
\hline Parameters & RF & FSO \\
\hline Range & $4 \mathrm{~km}$ & $4 \mathrm{~km}$ \\
\hline Capacity & Allowed & Not allowed \\
\hline Data rate & $100 \mathrm{Mb} / \mathrm{s}$ & $10 \mathrm{~Gb} / \mathrm{s}$ \\
\hline Spectrum range & $2-6 \mathrm{GHz}$ & $0.8-1.5 \mathrm{THz}$ \\
\hline Power & $2.31 \mathrm{E}-02(\mathrm{~J} / \mathrm{Mb})$ & $2.00 \mathrm{E}-03(\mathrm{~J} / \mathrm{Mb})$ \\
\hline Output power & $50 \mathrm{mWatt}$ & $5-500 \mathrm{mWatt}$ \\
\hline Power Loss & $5.7 \mathrm{GHz} 108 \mathrm{db} / \mathrm{km}$ & $5-15 \mathrm{db} / \mathrm{km}$ \\
\hline Security & Low & High \\
\hline Advantage & No line of sight & Unlicensed band \\
\hline Limitation & Spectrum & Environment \\
\hline
\end{tabular}

Instead of above mentioned range of radio frequency it also includes the range of frequency that is unlicensed band of $2.4 \mathrm{GHz}$. It varies from country to country as in some other countries range of $315 \mathrm{MHz}$ is used as unlicensed band of frequency. For unlicensed band the transmission becomes more sensitive is sue that it should not disturb the transmission of other non-licensed band users. So the users are also limited by the power and sensitivity. And also their data rate is kept in certain range for the transmission. Allowed transmitted power according to the rules of FCC is just $1 \mathrm{~W}$ att. Several other European countries have allowed less power than FCC allowed. The category of such devices is 802.11a. The data rate and received power sensitivity is shown in Fig. 8.

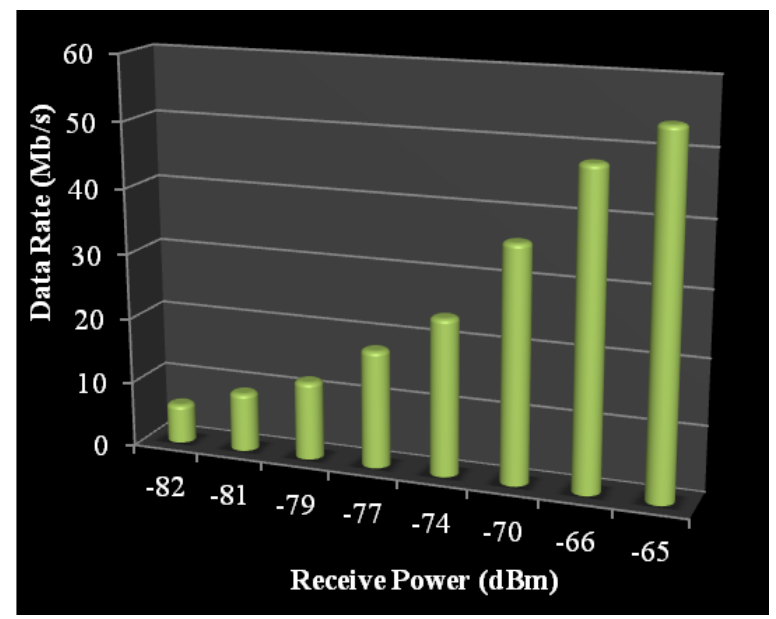

Fig.8. 802.11a Device data rate Vs received power

\section{H. FSO Data Security}

Free space optical technology uses different modulation techniques to transmit data in line of sight. Several researchers are doing research to transmit RF signal via free space optics in order to ensure the security and reliability. And there has been tremendous increase in the use of FSO technology due to the reason that all the bottlenecks of data capacity could be avoided. High speed internet is one of the major fields that have enhanced the use of FSO due to its virtually unlimited space. But this is no reason for any system to get success in the market user is very reluctant to adopt the technology that can't ensure his data security. The security level of any system involved from lower level to the higher level of security. So in designing the system of FSO security is maintained from physical layer to the networking protocol [19]. The major advantage of free space optics systems is that it does not involve any kind of broadcasting which is its major advantage over the radio frequency broadcasting system. So without being the physical interruption of any intruder it is not possible to catch the data passing through this technology. The equipment is deployed above the level of intrudes like vehicles and other obstacle so this value ensure the security of system. Birds or any other interruption can only momentarily damage the signal but still not very harmful for the system.

Another security concern of the system is diameter of 
beam to be tapped by any other intruder and for this purpose the beam width of the system could be narrow down to the limit that it could not be intercepted. This case is already achieved if the system is based on the laser technology. If free space optics is done by using the laser technology the beam with is already narrow and focused that if anyone will intervene into the system could be easily caught and the data security of the system could be ensured. If it considered that intruder has intercepted the signal than the receivers and transmitters are designed in such a way that whole of the system could go in failure mode and all of the remaining signals required for the data decryption would be totally lost.

Another aspect of security in free space optics is securing the connection of FSO system for the users. Transmitters and receivers are usually installed on the high rise buildings and the building sways. So for the transmitter and receiver to be synchronized in the communication the beam should be that much wider that with the movement of building they remain aligned with each their or could get enough of their signal to retrieve data. This type of secure communication for the short distance is good and helpful for user. But for the distant communication like few kilometers the beam width goes in meters and in this scenario beam width cause serious security issues. Because any intruder could get the signal by placing the mirror in the lin $\mathrm{k}$ and without disturbing the whole system could break the security of whole system. So small beam width are going to form more secure connection.

To overcome this security concern of free space optics system of communication designers have designed the system in such a way that a conical shape of beam is created in the field from transmitter to receiver and if any intruder places the beam tapping device to towards any side of transmitter or receiver would be caught so if the intruder place the device in exactly the middle of communication sight than security as got threat otherwise systemwould stop transmitting any data.

\section{RF Data Security}

It is of the most common interest of every communication is that data should remain secure from being hacked. RF system has increased the mobility and is becoming more popular over the years. The common characteristic of wireless communication is broadcasting of data over the air interface. Although the data is properly encoded and encrypted so that no one can breakout the information stored in it but the fact is that data is transmitted towards all the direction and any receiver operating and detecting this frequency can get the signal [11]. The example of this security breakout is in mobile communication any one can easily track the location of mobile number by just intercepting the data which is his personal property. Any outdated data encryption technique can harm the personal data security of the system.

Different application based on the wireless communication like Bluetooth and RFID system use RF links for communication. Some of the systems are reliable but some are not very authentic in terms of security and at any time their security could be breached. Different mobile phones and PDA's are used for the wireless communication and intent browsing so there is high risk of security. Although different coding and encryption schemes are used in the modern digital communication and these schemes are currently implemented in GSM, GPRS technologies. Over the air transmission it is very hard for any system to decode the messages. PN codes are used in data coding schemes and these PN codes are used when data is transmitted outside the network. The PN codes are assigned by the transmitter to bother transmitting schemes and receiver also know that PN code to decode the information stored in signals.

Due to the security concerns of broadcasting and point to point wireless communication different protocols are developed and these protocols are for every layer of the systems. The rules are imposed by the standard bodies and all telecommunication and radio frequency transmitting agencies are bound to follow these rules and protocols. So the standards introduced in the RF field are mentioned below:

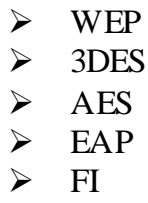

But still after implementing these standards some of these have been compromised in the field. Wireless equivalent privacy is the type of protocol that uses advanced coding techniques to encrypt the data. It uses RC4 algorith m and now banned in USA that its security has been breached. This protocol was developed for the wireless local area networking. And now they institutions have clearly given the instruction of making encryption schemes of wireless local area network to be compliant with FIPS 140-2. An unbreakable encryption scheme is 3DES but remain in the use for very short interval of time. It is advanced into the new form as AES.

\section{HYBRID RF/FSO SYSTEM}

Free space optical system came as new wireless technology to transmit data. It is certainly replacement of fiber optic technology in terms of its high bandwidth. Over the fiber optic technology it is time saving and could be rapidly deployed in the field. Another advantage is cost that is included in the fiber optic system is also reduced by this system [20,21]. RF technology is also very useful in terms of long distant communication where no line of sight is available. In some urban environment where there is long distant building, and it is not possible for FSO system to be completely installed in the network is backed by the radio frequency signaling. FSO lin $\mathrm{k}$ is disturbed by the fog in the weather conditions. The system is nearly unharmed with raining environment. RF links are heavily affected by the rain so these two mutual 
conditions developed the requirement of hybrid system.

Hybrid system architecture is comprised of mainly three subsystems as follows:

$>$ Laser Link

$>$ Switch

$>$ RF link

In normal situation data is transferred using the laser communication link. The receiver measure the data rate received after very short interval of time. When the conditions of laser link or optic link becomes down over a certain threshold and data rate drops from certain minimum level communication lin $\mathrm{k}$ is shifted from optic to radio frequency. There is no use of introducing more power in the system of laser signaling because harsh environmental conditions always degrade the signaling [22-25]. For shifting the system from laser or free space optics to radio frequency is done by the switch module that senses the signaling condition and switch over to other link. Now after switch the system the received signal strength of RF link also continuously monitored to again shift the system from RF to FSO as shown in Fig. 9.

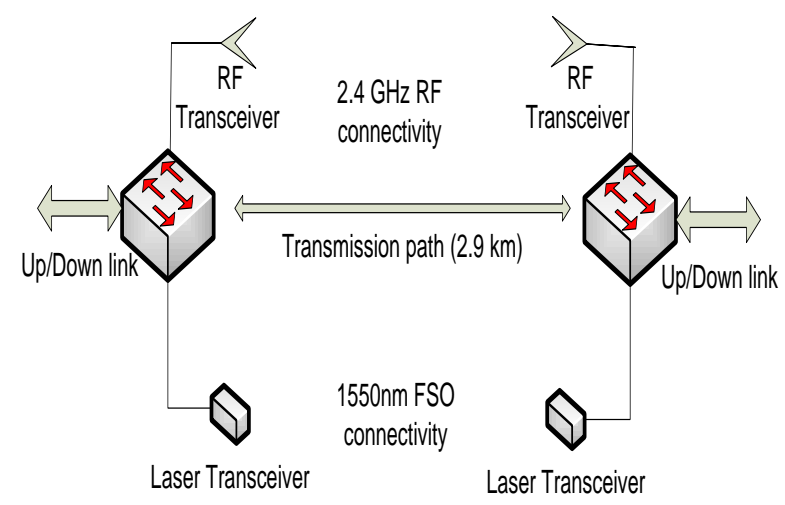

Fig.9. Basic hybrid RF/FSO architecture

In the hybrid system it is the matter of great concern that both individual systems should work at the same level for the greater throughput and higher efficiency. If in the system free space optics is working at $10 \mathrm{GHz}$ and $\mathrm{RF}$ is merely operating at mega hert $z$. Then the combined throughput would be limited to the RF system. So both systems should be operating at the same data rate for the hybrid system to work efficiently. To meet the carrierclass availability the link should be available for around 99.999\% of the time as shown in Fig. 10. FSO system caters all the requirement of communication condition but in only certain cases where long distance and harsh weather requirements have met it is RF that takes part into the communication. The hybrid system ensures the carrier class availability over the longer ranges. In many experiments hybrid RF and FSO systems has been studied and discussed for the maximum throughput capacity.

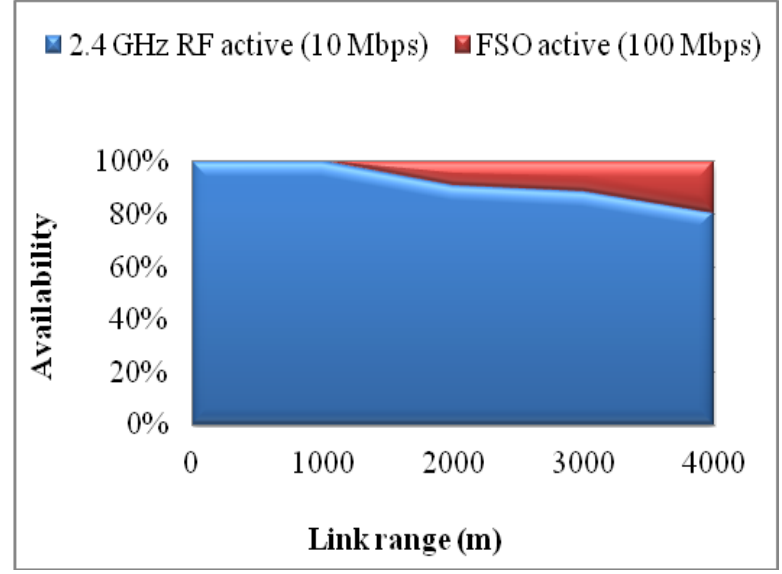

Fig.10. Hybrid system availability Vs link range

\section{CONCLUSION}

Free space technology is new for the world but growing at the rapid pace for the links that require higher data rate. The major benefit of the technology is noninterference with the RF links so both systems could be used independently as well as in combination at certain place to increase the efficiency of any system. Hybrid RF/FSO systems are used many fields where the requirement of weather conditions and distance are compensated for both of the technologies. In these scenarios greater throughput is achieved by the alternative use of both systems.

\section{REFERENCES}

[1] Z. Zhao, et al., "Radio frequency interference mitigation in OFDM based passive bistatic radar," AEU International Journal of Electronics and Communications, vol. 70, pp. 70-76, 2016.

[2] J. A. Lima, et al., "Analysis of Relay Attacks on RFiD Systems," Latin America Transactions, IEEE (Revista IEEE America Latina), vol. 10, pp. 1274-1282, 2012.

[3] H. Zhou, et al., "Optical power allocation for adaptive transmissions in wavelength-division multiplexing free space optical networks," Digital Communications and Networks, vol. 1, pp. 171-180, 2015.

[4] B. Mo, et al., "Line width analy sis of a tunable optical filter based on free-space optics," Optik - International Journal for Light and Electron Optics, vol. 125, pp. 6488 6490, 2014.

[5] C. Liu, et al., "Adaptive optics for the free-space coherent optical communications," Optics Communications, vol. 361, pp. 21-24, 2016.

[6] J. B. Rosolem, et al., "Optical sensing in high voltage transmission lines using power over fiber and free space optics," Optical Fiber Technology, vol. 26, Part B, pp. 180-183, 2015.

[7] A. Y. Davletbaev, et al., "Multi-stage hydraulic fracturing and radio-frequency electromagnetic radiation for heavyoil production," Journal of Unconventional Oil and Gas Resources, vol. 12, pp. 15-22, 2015. 
[8] R. Yu-Jiun, et al., "High performance multiband radio antenna," in Antennas and Propagation \& USNC/URSI National Radio Science Meeting, 2015 IEEE International Symposium on, 2015, pp. 928-929.

[9] T. Aboufoul, et al., "Reconfiguring UWB Monopole Antenna for Cognitive Radio Applications Using GaAs FET Switches," Antennas and Wireless Propagation Letters, IEEE, vol. 11, pp. 392-394, 2012.

[10] H. A. Fadhil, et al., "Optimization of free space optics parameters: An optimum solution for bad weather conditions," Optik - International Journal for Light and Electron Optics, vol. 124, pp. 3969-3973, 2013.

[11] K. Prabu, et al., "Spectrum analysis of radio over free space optical communications systems through different channel models," Optik - International Journal for Light and Electron Optics, vol. 126, pp. 1142-1145, 2015.

[12] Bakhsh, S. T., et al. "Self-Schedule and Self-Distributive MAC Scheduling Algorithms for Next-Generation Sensor Networks." International Journal of Distributed Sensor Networks, pp. 1-15, 2015.

[13] N. Kumar and A. K. Rana, "Impact of various parameters on the performance of free space optics communication system," Optik - International Journal for Light and Electron Optics, vol. 124, pp. 5774-5776, 2013.

[14] F. E. Zocchi, "A simple analytical model of adaptive optics for direct detection free-space optical communication," Optics Communications, vol. 248, pp. 359-374, 2005.

[15] L. Hou, et al., "Radio frequency heating for postharvest control of pests in agricultural products: A review," Postharvest Biology and Technology, vol. 113, pp. 106$118,2016$.

[16] Z. Li, et al., "Combinational-deformable-mirror adaptive optics sy stem for atmospheric compensation in free space communication," Optics Communications, vol. 320, pp. 162-168, 2014.

[17] I. Mitsuishi, et al., "Ray-tracing simulations for the ultralightweight X-ray optics toward a future jupiter exploration mission," Advances in Space Research, vol. 57, pp. 320-328, 2016.

[18] G. Aldabbagh, et al., "Distributed dynamic load balancing in a heterogeneous network using LTE and TV white spaces," Wireless Networks, pp. 1-12, 2015.

[19] A. M. Rincon and R. K. Singh, "Inactivation of Shiga toxin-producing and nonpathogenic Escherichia coli in non-intact steaks cooked in a radio frequency oven," Food Control, vol. 62, pp. 390-396, 2016.

[20] J. Shi, et al., "A tumor-specific cleavable nanosystem of PEG-modified C60@Au hybrid aggregates for radio frequency-controlled release, hy perthermia, photody namic therapy and X-ray imaging," Acta Biomaterialia, vol. 29, pp. 282-297, 2016.

[21] M. Usman, et al., "Performance Analysis of Switching Based Hybrid FSO/RF Transmission," in Vehicular Technology Conference (VTC Fall), 2014 IEEE 80th, 2014, pp. 1-5.

[22] I. B. Djordjevic and G. T. Djordjevic, "On the high-speed communication over hybrid free-space optical (FSO) wireless fading channels," in LEOS Annual Meeting Conference Proceedings, 2009. LEOS '09. IEEE, 2009, pp 833-834.

[23] G. Aldabbagh, et al., "QoS-Aware Tethering in a Heterogeneous Wireless Network using LTE and TV White Spaces," Computer Networks, vol. 81, pp. 136-146, 2015.

[24] Nisar A. Lala, et al., "Novel Spectrum Handoff in Cognitive Radio Networks Using Fuzzy Logic",
International Journal of Information Technology and Computer Science (IJITCS), vol. 5, pp. 103-110, 2013.

[25] V. Jayaraj, J, et al., "Impact of Throughput in Enhancing the Efficiency of Cognitive Radio Ad Hoc Network - A Study", International Journal of Information Technology and Computer Science (IJITCS), vol. 5, pp. 70-77, 2013.

\section{Authors' Profiles}

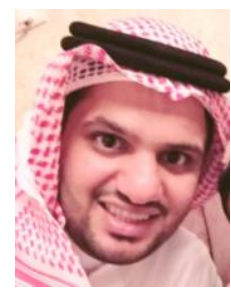

Rayan Atteah Alsemmeari received the Master degree from Western Michigan University, USA in 2012. He joined the Department of Computer Sciences, King Abdulaziz University in 2008 and working as lecturer. During Alsemmeari master study at WMU he worked on different wireless network projects. His research interests are mainly in the area of VOIP, FSO, RF, wireless communication, information retrieval, Data mining, and Mobile ad hoc network.

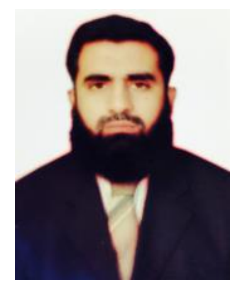

Dr. Sheikh Tahir Bakhsh has been bestowed upon by Gold Medal by the Rector COMSATS Institute of Information Technology, Abbottabad, Pakistan for securing 1st position in MCS in August 2006. Dr. Tahir has received the Ph.D. degree in Computer and Information Sciences from Universiti Teknologi PETRONAS, Malaysia in 2012. He joined the faculty of Computing and Information Technology, King Abdul Aziz University, Saudi Arabia as an Assistant professor in 2013. In the recent he has completed LTE HICI project with the collaboration of Stanford. He has also directed graduate and undergrad graduate projects. His areas of reach interests include Bluetooth network, Wireless sensor network (WSN), Mobile ad hoc network (MANET), and Computer networks. He works mainly on wireless network protocol designs optimizing the performance of networks. Recently, he has been involved in project related physical protocol design for Bluetooth scatternet. $\mathrm{He}$ has published more than 25 journal articles and referred conference papers in these areas.

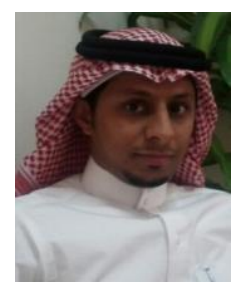

Hani Alsemmeari has been a faculty trainer and a consultant at the Institute of Public Administration (IPA), Saudi Arabia (SA), since 2007. Mr. Alsemmeari has received his bachelor degree in Computer Science (CS) in 2005 from King Abdulaziz University, SA, and got his master degree in CS from Western Michigan University, USA in 2010. Mr. Alsemmeari's research interests include: Cloud computing, Databases, Data Mining, Semantic Web and Web Programming

How to cite this paper: Rayan A. Alsemmeari, Sheikh Tahir Bakhsh, Hani Alsemmeari, "Free Space Optics Vs Radio Frequency Wireless Communication", International Journal of Information Technology and Computer Science (IJITCS), Vol.8, No.9, pp.1-8, 2016. DOI: 10.5815/ijitcs.2016.09.01 